\title{
Pitfall of ${ }^{18}$ F-FDG PET/CT in Characterization of Relapsed Multisystem Lymphomatoid Granulomatosis
}

\author{
Kelly Tung ${ }^{1}$, Allison C. Rosenthal ${ }^{2}$, Fiona E. Craig ${ }^{3}$, Jonathan B. Ashman ${ }^{4}$, and Ming Yang ${ }^{1}$ \\ ${ }^{1}$ Department of Radiology, Mayo Clinic Arizona, Scottsdale, Arizona; ${ }^{2}$ Department of Hematology/Oncology, Mayo Clinic Arizona, \\ Scottsdale, Arizona; ${ }^{3}$ Department of Laboratory Medicine and Pathology, Mayo Clinic Arizona, Scottsdale, Arizona; and \\ ${ }^{4}$ Department of Radiation Oncology, Mayo Clinic Arizona, Scottsdale, Arizona
}

\begin{abstract}
We present serial ${ }^{18} \mathrm{~F}-\mathrm{FDG}$ PET/CT findings in a case of grade 3 pulmonary lymphomatoid granulomatosis positive for the EpsteinBarr virus. The patient experienced a transient complete response to R-CHOP chemotherapy and subsequent multisystem recurrence, predominately involving the subcutaneous region of the torso on ${ }^{18} \mathrm{~F}-\mathrm{FDG} \mathrm{PET} / \mathrm{CT}$. Biopsy of the most hypermetabolic subcutaneous lesion demonstrated grade 1 cutaneous lymphomatoid granulomatosis negative for the Epstein-Barr virus. This report highlights the role of ${ }^{18} \mathrm{~F}-\mathrm{FDG}$ PET/CT in characterizing and monitoring disease progression and regression, as well as the limitations of ${ }^{18} \mathrm{~F}-\mathrm{FDG}$ PET/CT in accurate grading of multisystem recurrence, given the diversity of clinical and histopathologic features of lymphomatoid granulomatosis.
\end{abstract}

Key Words: hematology; lymphoma; PET/CT; ${ }^{18}$ F-FDG; lung; lymphomatoid granulomatosis

J Nucl Med Technol 2018; 46:396-397

DOI: 10.2967/jnmt.118.212274

$\mathbf{L}$

ymphomatoid granulomatosis is a rare T-cell rich, Epstein-Barr virus associated B-cell lymphoproliferative disorder, predominately involving the lung (1). Accurate grading information on this abnormality is essential in determining clinical management and prognosis. ${ }^{18} \mathrm{~F}-\mathrm{FDG}$ PET/CT plays an important role in staging lymphomatoid granulomatosis, in monitoring therapeutic response, and in guiding biopsy (2).

\section{CASE REPORT}

A 65-year-old man presented with multiple pulmonary nodules and underwent left lower lobe wedge resection. Histopathologic examination confirmed lymphomatoid granulomatosis of grade 3 on the World Health Organization classification, with atypical large B cells positive for Epstein-Barr virus. A staging ${ }^{18} \mathrm{~F}$-FDG PET/CT scan showed multiple hypermetabolic nodules in both lungs (Fig. 1A). The patient received

Received Apr. 2, 2018; revision accepted May 31, 2018.

For correspondence contact: Ming Yang, Mayo Clinic Arizona, $13400 \mathrm{E}$. Shea Blvd., Scottsdale, AZ 85259.

E-mail: yang.ming@mayo.edu

Published online Jun. 8, 2018.

COPYRIGHT (c) 2018 by the Society of Nuclear Medicine and Molecular Imaging.
6 cycles of rituximab, cyclophosphamide, doxorubicin, vincristine, and prednisone (R-CHOP) chemotherapy. Posttherapeutic ${ }^{18}$ F-FDG PET/CT showed resolution of all lung lesions, indicating a complete response to therapy (Fig. 1B). Seven months later, the patient presented with left ear pain and multiple subcutaneous nodules. A restaging ${ }^{18} \mathrm{~F}-\mathrm{FDG}$ PET/CT scan demonstrated numerous hypermetabolic subcutaneous nodules in the torso and a hypermetabolic nodule in the left lung (Fig. 1C). Brain MRI showed an enhancing left-trigeminalnerve lesion, which was also ${ }^{18} \mathrm{~F}-\mathrm{FDG}$ avid (Figs. $2 \mathrm{~A}-2 \mathrm{C}$ ) and was presumed to represent involvement of the lymphomatoid granulomatosis in the central nervous system. Excisional biopsy of the most ${ }^{18} \mathrm{~F}-\mathrm{FDG}$ avid subcutaneous nodule in the right anterior chest (Figs. 3A and 3B) demonstrated grade 1 lymphomatoid granulomatosis negative for the Epstein-Barr virus. The patient declined lung biopsy and additional chemotherapy. Palliative radiation therapy was delivered for symptomatic trigeminal neuralgia.

\section{DISCUSSION}

Lymphomatoid granulomatosis predominantly affects the lungs $(>90 \%)$, followed by the skin $(25 \%-50 \%)$, the kidneys $(32 \%-40 \%)$, and the central nervous system $(20 \%-30 \%)(1)$.

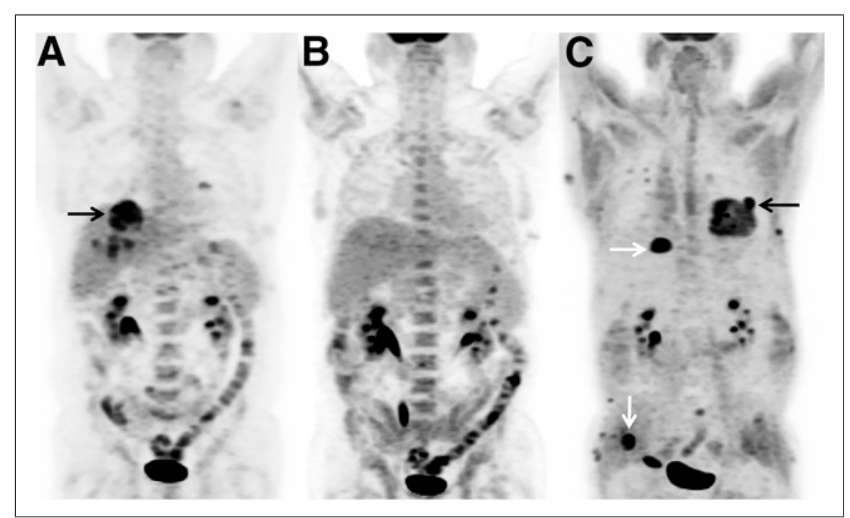

FIGURE 1. (A) Staging 18F-FDG PET/CT showed multiple hypermetabolic lung lesions (arrow). (B) After 6 cycles of RCHOP therapy, all lesions resolved. (C) Seven months later, restaging ${ }^{18} \mathrm{~F}-\mathrm{FDG} \mathrm{PET} / \mathrm{CT}$ showed multiple subcutaneous hypermetabolic lesions (white arrows) and a left lung hypermetabolic nodule (black arrow). 


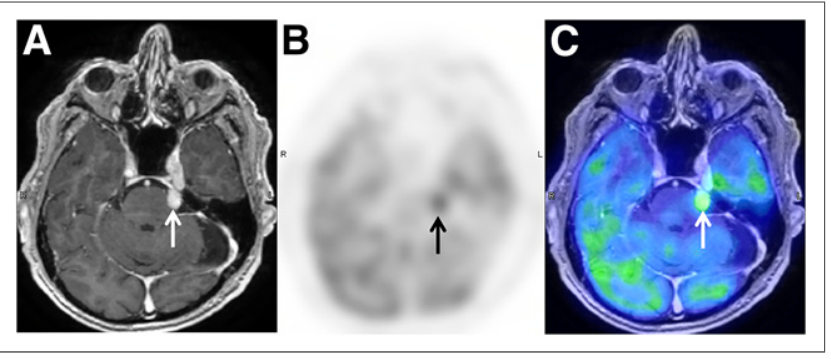

FIGURE 2. Contrast-enhanced brain MRI showed enhancing lesion (arrows) involving left trigeminal nerve $(A)$, which was ${ }^{18} \mathrm{~F}-$ FDG avid (SUV max, 6.3$)$ on restaging ${ }^{18}$ F-FDG PET (B) and PET/ MRI (C).

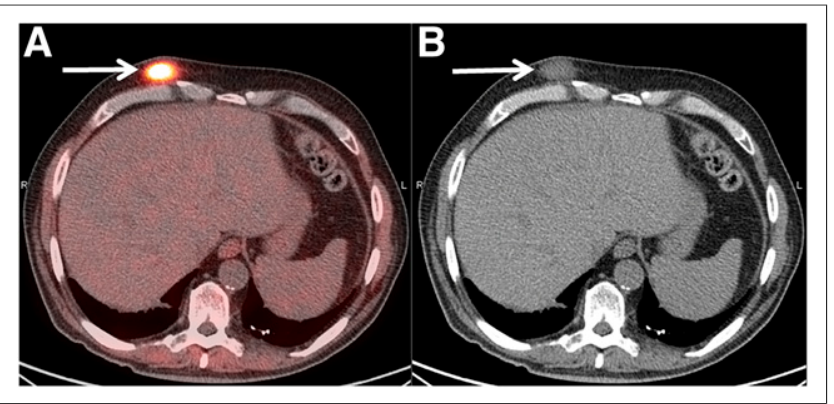

FIGURE 3. Restaging ${ }^{18} \mathrm{~F}-\mathrm{FDG}$ PET/CT showed ${ }^{18} \mathrm{~F}-\mathrm{FDG}$ avid subcutaneous nodule (arrows) with SUV $\mathrm{max}_{\max }$ of 10.3 on right anterior chest wall. Excisional biopsy confirmed grade 1 cutaneous lymphomatoid granulomatosis.

The clinical behavior of lymphomatoid granulomatosis is highly variable, with approximately $12 \%$ of cases progressing to malignant lymphoma. Chemotherapy regimens for diffuse large B-cell lymphoma, such as R-CHOP, have shown promise for high-mortality grade 3 pulmonary lymphomatoid granulomatosis (3).
Histopathologic diagnosis of lymphomatoid granulomatosis relies on the cytologic atypia and density of clonal B cells positive for Epstein-Barr virus from the lung specimen (1). Of the few reported cases of concomitant pulmonarycutaneous lymphomatoid granulomatosis, up to half showed absence of the Epstein-Barr virus, indicating discordant grading between the skin lesions and the lung lesions $(4,5)$. In the current case, therefore, grading based on histopathologic examination of the cutaneous specimen was not reliable in predicting the clinical course or in guiding management.

\section{CONCLUSION}

${ }^{18}$ F-FDG PET/CT is a useful imaging tool in the surveillance of lymphomatoid granulomatosis but lacks accuracy in grading lymphomatoid granulomatosis, given the variability of Epstein-Barr virus positivity and the diversity of clinical features in cutaneous lesions. Histopathologic examination of lung lesion specimens is the reliable grading method in relapsed multisystem lymphomatoid granulomatosis.

\section{DISCLOSURE}

No potential conflict of interest relevant to this article was reported.

\section{REFERENCES}

1. Liebow AA, Carrington CR, Friedman PJ. Lymphomatoid granulomatosis. Hum Pathol. 1972;3:457-558.

2. Roarke MC, Nguyen BD. PET/CT characterization and monitoring of disease activity in lymphomatoid granulomatosis. Clin Nucl Med. 2007;32:258-259.

3. Polizzotto MN, Dawson MA, Opat SS. Failure of rituximab monotherapy in lymphomatoid granulomatosis. Eur J Haematol. 2005;75:172-173.

4. Messana K, Marburger T, Bergfeld W. EBV-negative cutaneous lymphomatoid granulomatosis with concomitant EBV-positive pulmonary involvement: a potential diagnostic and prognostic pitfall. Am J Dermatopathol. 2015;37:707-711.

5. Beaty MW, Toro J, Sorbara L, et al. Cutaneous lymphomatoid granulomatosis: correlation of clinical and biologic features. Am J Surg Pathol. 2001;25:11111120 . 